\title{
O Cadáver no Ensino da Anatomia Humana: uma Visão Metodológica e Bioética
}

\author{
The Corpse in the Teaching of Human \\ Anatomy: a Methodological and Bioethics \\ Overview
}

\author{
Gilliene Batista Ferreira da Costa ${ }^{I}$ \\ Gilliane Batista Ferreira da Costa \\ Carla Cabral dos Santos Accioly Lins ${ }^{I}$
}

\section{- Anatomia. \\ - Metodologia. \\ - Bioética. \\ - Ensino.}

PALAVRAS-CHAVE
Aprovado em: 07/02/2012

Reencaminhado em: 07/08/2012

Aprovado em: 13/08/2012

\section{ABSTRACT}

This study aimed to analyze the opinion of students about the teaching methodologies applied in their practical classes of human anatomy, as well as to approach bioethical issues involved in the use of cadavers. This study was conducted through a questionnaire applied to 542 students of courses at the Health Sciences Center, Federal University in Pernambuco, who were attending the course of human anatomy in 2011. The data obtained showed that the use of human cadaver in practical classes was considered essential by $88.9 \%$ of surveyed, and $98.3 \%$ use some auxiliary teaching material. As for the bioethical issues, $80.7 \%$ said they had received information regarding the handling a corpse. Thus we see that the use of human corpses was considered essential to the process of teaching and learning in the study of human anatomy, and that the handling of cadavers reflect in the conduct of the future professional with their patients, strengthening the humanization of health professionals. 


\section{INTRODUÇÃO}

A anatomia humana é uma disciplina básica para todos os estudantes ingressantes na área da saúde ${ }^{1,2}$. Nela, os alunos aprendem a forma e a localização das estruturas do corpo humano, correlacionando-as com suas funções ${ }^{3}$.

Para que este conhecimento se concretize, são aplicadas diversas metodologias, dentre elas o uso de cadáveres humanos dissecados, que representam a forma mais antiga e uma das mais utilizadas ainda nos dias de hoje para o ensino da anatomia humana ${ }^{4}$. Associados a eles, diversos recursos auxiliam no processo de ensino-aprendizagem, como o uso de peças anatômicas de plástico e meios eletrônicos ${ }^{5}$. Recentemente, incluiu-se o processo de aprendizagem autodirigido baseado em problemas, com acesso individual do aluno ao laboratório morfofuncional e de informática ${ }^{6}$.

Essas novas ferramentas conduzem à melhoria de desempenho do aluno, já que facilitam o aprendizado de conteúdos considerados difíceis de compreender por meio de aulas expositivas $^{7}$, permitindo a construção efetiva do conhecimento ${ }^{8}$. Diante da diminuição do número de doações de cadáveres, os métodos alternativos de ensino na anatomia tornam-se cada vez mais úteis, visto que a necessidade de corpos para estudo é maior do que a disponibilidade ${ }^{4}$.

Do ponto de vista bioético, o cadáver humano não deve ser visto como simples objeto de estudo, já que é envolvido por um vínculo emocional e afetivo com os indivíduos com que estabeleceu uma relação. A questão da morte está presente desde o início do ensino médico e das outras profissões da área da saúde, sendo inegável que o avanço da medicina se deu graças à possibilidade de ensino e pesquisa ética em cadáveres 9 . No entanto, opiniões diversas são encontradas atualmente quanto à vantagem de usar peças cadavéricas no ensino da anatomia humana.

Alguns apontam repulsa visual e forte odor de formol como aspectos negativos no uso do cadáver para ensino, os quais podem constituir uma barreira importante ao aprendizado $^{3}$. Quanto ao lado positivo, defende-se que a ausência de cadáveres para estudo compromete o aproveitamento dos alunos de todos os cursos da área da saúde ${ }^{2}$, com enfraquecimento do processo de humanização ${ }^{10}$.

Dessa forma, é preciso vincular os aspectos técnicos aos ético-humanísticos para que os estudantes possam desenvolver habilidades para a competência em suas profissões sem que se esqueçam do lado humanístico do assunto ${ }^{11}$.

Mitre et al. ${ }^{12}$ afirmaram que um dos méritos da educação atual consiste na

[...] crescente tendência à busca de métodos inovadores, que admitam uma prática pedagógica ética, crítica, re- flexiva e transformadora, ultrapassando os limites do treinamento puramente técnico, para efetivamente alcançar a formação do homem como um ser histórico, inscrito na dialética da ação-reflexão-ação. (p. 2134)

Os objetivos deste estudo foram relatar a opinião dos estudantes sobre as metodologias de ensino utilizadas nas atividades práticas da anatomia humana e abordar questões bioéticas que envolvem o manuseio do cadáver humano.

\section{MATERIAL E MÉTODOS}

Foi realizado um estudo descritivo, baseado em levantamento de dados mediante aplicação de questionários que continham questões exclusivamente objetivas relacionadas às metodologias aplicadas no ensino da anatomia humana, assim como questões bioéticas ligadas ao uso de cadáveres humanos para estudo. Este trabalho foi aprovado pelo Comitê de Ética em Pesquisa Envolvendo Seres Humanos do Centro de Ciências da Saúde da Universidade Federal de Pernambuco (CAAE 0406.0.172.000-11)

A pesquisa foi realizada com 542 estudantes que cursavam a disciplina de anatomia humana da Universidade Federal de Pernambuco, dos cursos do Centro de Ciências da Saúde (Medicina, Odontologia, Fisioterapia, Fonoaudiologia, Educação Física, Farmácia, Terapia Ocupacional e Nutrição), do segundo semestre letivo do ano de 2011, que deram seu consentimento após a leitura do termo de consentimento livre e esclarecido.

Quanto ao tema metodologia, os alunos foram questionados sobre: se em suas aulas práticas utilizavam-se cadáveres para estudo; se era indispensável o seu uso e se eles poderiam ser substituídos por programas de computador, material sintético ou animais, assim como se deveriam ser utilizados somente para pesquisa; quais eram os materiais didáticos auxiliares que eles empregavam e se seus professores faziam uso de alguma tecnologia em sala de aula. Quanto ao assunto bioética, questionou-se acerca de: se eles haviam recebido algum tipo de informação sobre a importância do respeito ao manipular um cadáver; se possuir coragem e maturidade emocional era necessário para manipulá-lo, assim como a influência religiosa; se as aulas de anatomia humana preparam o futuro profissional para ter equilíbrio emocional.

\section{RESULTADOS E DISCUSSÃO}

Dos 542 estudantes que responderam ao questionário, 342 $(63,1 \%)$ foram do gênero feminino e 200 (36,9\%) do masculino, obtendo-se uma proporção feminino:masculino de 1,7:1,0. A média de idade foi de 20,15 anos, com idade mínima registra- 
da de 16 anos e máxima de 58 anos. Os alunos pertenciam a diversos cursos da área de saúde: Medicina, Odontologia, Fisioterapia, Fonoaudiologia, Educação Física, Farmácia, Terapia Ocupacional e Nutrição (Figura 1).

FIGURA 1

Distribuição dos estudantes pesquisados por curso

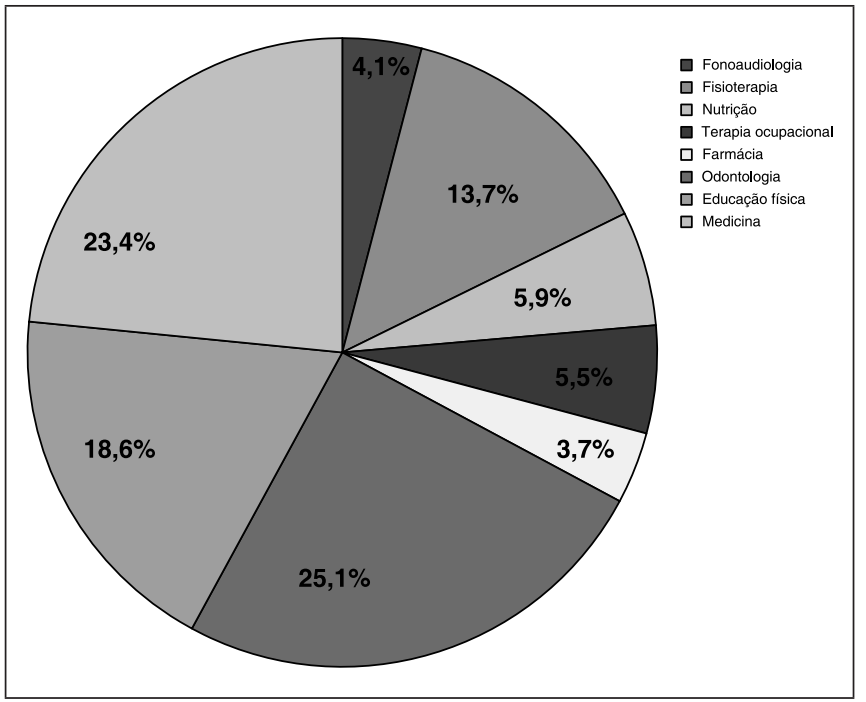

Fonte: Departamento de Anatomia Humana/UFPE

Quando questionados sobre religião, 287 (55,6\%) afirmaram ser católicos, 125 (24,2\%) evangélicos, 46 (8,9\%) espíritas, $30(5,8 \%)$ disseram não ter nenhuma religião e $28(5,4 \%)$ seguiam outras religiões. Do total dos pesquisados, $18,6 \%$ consideraram que a religião influencia sua conduta frente à manipulação de um cadáver.

Todos os estudantes afirmaram utilizar cadáveres humanos para estudo em suas aulas práticas na universidade. Desse universo, $482(88,9 \%)$ consideraram seu uso indispensável nas aulas de anatomia (Tabela 1). Esses cadáveres vêm sendo utilizados pelos alunos apenas para a demonstração das estruturas anatômicas, já que, segundo Melo e Pinheiro ${ }^{5}$, a redução na doação de cadáveres fez com que os alunos do curso médico da Universidade Federal de Pernambuco deixassem de dissecar desde o ano de 1998. Ainda segundo esses autores, a dificuldade de entrada de cadáveres no Departamento de Anatomia Humana dessa universidade tem feito com que as aulas práticas sejam realizadas com cadáveres dissecados várias vezes, alguns até mesmo com objetivos inexistentes.

Quando perguntados se o cadáver humano deveria ser utilizado somente nas pesquisas científicas e não em aulas de graduação, 517 (95,7\%) estudantes não concordaram. Quando questionados se o uso de cadáveres poderia ser substituído por programas de computadores e materiais sintéticos, 443 $(81,9 \%)$ dos alunos responderam negativamente. E quando interrogados sobre a substituição por animais, 530 (98,1\%) responderam que não são a favor. Segundo Aversi-Ferreira et al. ${ }^{13}$, a dissecação de cadáveres na anatomia humana é uma ferramenta importante para a educação médica, por ser uma metodologia de ensino que encoraja o pensamento crítico e investigativo, integrando o ensino básico aos conhecimentos clínicos. Dessa forma, mesmo sem realizarem a dissecação de cadáveres humanos, utilizando-os apenas para observação das estruturas anatômicas, comprometendo o desenvolvimento das habilidades para a formação médica, os estudantes expressaram a necessidade de ao menos ter contato com os cadáveres em suas aulas práticas.

TABELA 1

Opinião dos estudantes de diferentes cursos quando questionados se consideram indispensável o uso de cadáver humano nas aulas práticas de anatomia

\begin{tabular}{lcl}
\hline \multicolumn{1}{c}{ Curso } & Sim (\%) & Não (\%) \\
\hline Fonoaudiologia & $18(81,8 \%)$ & $4(18,2 \%)$ \\
Fisioterapia & $71(95,9 \%)$ & $3(4,1 \%)$ \\
Nutrição & $27(84,4 \%)$ & $5(15,5 \%)$ \\
Terapia ocupacional & $27(90 \%)$ & $3(10 \%)$ \\
Farmácia & $20(100 \%)$ & - \\
Odontologia & $115(84,6 \%)$ & $21(15,4 \%)$ \\
Educação física & $91(90,1 \%)$ & $10(9,9 \%)$ \\
Medicina & $113(89 \%)$ & $14(11 \%)$ \\
\hline
\end{tabular}

Fonte: Departamento de Anatomia Humana/UFPE.

Questionados sobre a utilização de materiais didáticos para auxiliar seu aprendizado, 98,3\% afirmaram fazer uso, sendo que 476 (87,8\%) declararam utilizar o atlas, $113(20,8 \%)$ os modelos de estudo e 131 (24,2\%) os programas de computador. A internet foi citada por 413 (76,2\%) alunos, sendo o recurso tecnológico mais utilizado atualmente em estudo a distância e se apresentando como um recurso adicional para professores, alunos e profissionais de saúde, tornando a educação mais interativa e viável ${ }^{14}$. Todos esses materiais didáticos de auxílio à aprendizagem, se usados de forma correta, contribuem para a compreensão do funcionamento correto dos órgãos do corpo e para a construção de conhecimentos ${ }^{15}$.

Os professores de anatomia humana da universidade pesquisada também foram avaliados pelos estudantes quanto ao uso de novas tecnologias nas aulas teóricas ou práticas. Dos alunos pesquisados, 36,2\% afirmaram que seus professores fazem uso de novas tecnologias. Esses dados são relevantes, já 
que pesquisas demonstram que aulas meramente expositivas, nas quais os alunos apenas veem e ouvem, são menos eficientes quando comparadas àquelas em que o aluno vê, ouve, interage e executa ${ }^{3}$.

A constante evolução da tecnologia educacional faz com que os professores precisem adaptar os métodos de ensino-aprendizagem para inserir o aluno em seu contexto diário. Isso leva o professor a se posicionar de forma receptiva às transformações e às avaliações que contribuem para o aprimoramento metodológico, muitas vezes aperfeiçoando o domínio de tecnologias modernas ${ }^{14,16}$.

$\mathrm{Na}$ abordagem das questões bioéticas, 434 estudantes $(80,7 \%)$ afirmaram ter recebido alguma informação sobre a importância do respeito ao manipular um cadáver ou parte dele, não o tratando como um simples material de estudo. Esse resultado pode ser consequência, muitas vezes, da diversidade cultural, religiosa e biográfica dos estudantes, o que faz com que o trabalho de conscientização realizado antes das primeiras aulas práticas não atinja a totalidade dos alunos ${ }^{17}$.

Maturidade emocional foi tida como uma condição essencial para se adotar uma postura adequada diante de um cadáver por 519 alunos (96,3\%). Já 366 (68\%) deles afirmaram que, para seguir carreira na área das Ciências da Saúde, o indivíduo deve ter, obrigatoriamente, coragem de ver um cadáver ou manipulá-lo.

Os pesquisados $(74,6 \%)$ consideram ainda que as aulas de anatomia humana preparam o futuro profissional para ter equilíbrio emocional e ser mais humano. Essa opinião vai ao encontro daqueles que sustentam que a ausência da relação do aluno com o cadáver implica um futuro enfraquecimento da relação com o paciente, dificultando o processo da humanização ${ }^{10}$.

As escolas médicas ainda enfrentam dificuldades para assumir a temática da morte com seus alunos, ficando a questão do morrer ligada apenas ao aspecto técnico, deixando-se de relacioná-la com o conteúdo de humanização que a cerca. $\mathrm{O}$ envolvimento do estudante com a morte durante a graduação fortalece a relação médico-paciente durante a vida profissional. O diálogo sobre a morte no processo de construção do "ser médico" contribui para melhorar a qualidade de vida e de morte presente na prática médica ${ }^{18}$. Para os que entram em contato com o cadáver em sua profissão, demonstra-se que há uma valoração da pessoa, ainda que representada em um corpo sem vida ${ }^{19}$.

\section{CONCLUSÃO}

O uso de cadáveres humanos, mesmo que apenas para demonstração das estruturas anatômicas, foi considerado indispensável ao processo de ensino-aprendizagem no estudo da anatomia humana. O manuseio do cadáver pelo estudante foi tido como uma forma de fortalecer a humanização dos futuros profissionais da saúde, refletindo-se em suas condutas com os pacientes.

\section{REFERÊNCIAS}

1. Siqueiro Neto EGB, Ferreira JR. O ensino da anatomia humana no curso de medicina da Universidade Federal de Goiás - avaliação e perspectivas. Arq Ciênc Saúde Unipar. 2001; 5(1):41-50.

2. Kruse MHL. Anatomia: a ordem do corpo. Rev Bras Enferm. 2004; 57(1):79-84.

3. Braz PRP. Método didático aplicado ao ensino da anatomia humana. Anuário da produção acadêmica docente. 2009; 3(4):303-310.

4. Costa LF, Feijós AGS. Doação de corpos: estudo comparativo luso-brasileiro sobre a utilização do corpo humano para ensino e pesquisa. Anais do $5^{\circ}$ Salão de Iniciação Científica; 2009. Porto Alegre: EDIPUCRS; 2009.

5. Melo EM, Pinheiro JT. Procedimentos legais e protocolos para utilização de cadáveres no ensino de anatomia em Pernambuco. Rev Bras Educ Med. 2010; 34(2):315-323.

6. Gomes R, Francisco AM, Tonhom SFR, Costa MCG, Hamamoto CG, Pinheiro OL, Moreira HM, Hafner MLMB. A formação médica ancorada na aprendizagem baseada em problema: uma avaliação qualitativa. Interface comunic saúde educ. 2009; 13(28):71-83.

7. Nunes EPS, Nunes FLS, Tori R. Avaliação da aquisição de conhecimento em ambientes virtuais de aprendizagem 3D para treinamento médico. XXXI Congresso da Sociedade Brasileira de Computação; 2011 jul. 19-22; Natal-RN, Brasil. Disponível em: http://www.dimap.ufrn.br/csbc2011/ anais/eventos/contents/WIM/WIM_Sessao_4_Artigo_6_Nunes.pdf.

8. Mota MF, Mata FR, Aversi-Ferreira TA. Constructivist pedagogic method used in the teaching of human anatomy. Int J Morphol. 2010; 28(2):369-374.

9. Cohen C, Gobbetti G. Bioética e morte: respeito aos cadáveres. Rev Assoc Med Bras. 2003; 49(2): 117-36.

10. Queiroz CAF. O uso de cadáveres humanos como instrumento na construção de conhecimento a partir de uma visão bioética. Goiás; 2005. Mestrado [Dissertação] — Universidade Católica de Goiás.

11. Gomes AP, Rego S, Palácios M, Siqueira-Batista R. Análise bioética do uso de recém-cadáveres na aprendizagem prática em medicina. Rev Assoc Med Bras. 2010; 56(1):11-6.

12. Mitre SM, Siqueira-Batista R, Girardi-de-Mendonça JM, Morais-Pinto NM, Meirelles CAB, Pinto-Porto C, Moreira 
T, Hoffman LMA. Metodologias ativas de ensino-aprendizagem na formação profissional em saúde: debates atuais. Cien Saude Colet. 2008; 13:2133-2144.

13. Aversi-Ferreira TA, Nascimento GNL, Vera I, Lucchese R. The practice of dissection as teaching methodology in anatomy applied to medical education. Int J Morphol. 2010; 28(1):265-272.

14. Piazza BL, Reppold Filho AR. O ensino de anatomia humana nos cursos de Educação Física da região metropolitana de Porto Alegre. Ciência em Movimento. 2011; 26:99-109.

15. Bucarey S, Alvarez L. Metodologia de construccion de objetos de aprendizaje para La ensenanza de anatomia humana em cursos integrados. Int. J Morphol. 2006; 24(3):357-362.

16. Fornaziero CC, Gordan PA, Carvalho MAV, Araújo JC, Aquino JCB. O Ensino da Anatomia: Integração do Corpo Humano e Meio Ambiente. Rev Bras Educ Med. 2010; 34(2):290-297.

17. Ruiz CR, Pessini L. Lições de anatomia: vida, morte e dignidade. O mundo da saúde. 2006; 30(3):425-433.

18. Silva GSN. A construção do "ser médico" e a morte: significados e implicações para a humanização do cuidado. São Paulo; 2006. Doutorado [Tese] — Faculdade de Medicina da Universidade de São Paulo.

19. Köhler ICC. O cotidiano dos funcionários de um instituto médico legal frente à morte: uma leitura à partir da bioéti- ca personalista. São Paulo; 2006. Doutorado [Tese] — Faculdade de Odontologia da Universidade de São Paulo.

\section{CONTRIBUIÇÃO DOS AUTORES}

Gilliene Batista Ferreira da Costa contribuiu na concepção e desenho do estudo, coleta de dados, análise e interpretação dos dados, elaboração do artigo e aprovação final da versão encaminhada para publicação. Gilliane Batista Ferreira da Costa contribuiu na coleta de dados, análise e interpretação dos dados, revisão crítica do artigo e na aprovação final da versão encaminhada para publicação. Carla Cabral dos Santos Accioly Lins contribuiu na concepção e desenho do estudo, coleta de dados, revisão crítica do artigo e aprovação final da versão encaminhada para publicação.

\section{CONFLITO DE INTERESSES}

Declararam não haver.

\section{ENDEREÇO PARA CORRESPONDÊNCIA}

Gilliene Batista Ferreira da Costa

Av. Conde da Boa Vista, 1482 — apto 111

Boa Vista - Recife

CEP 50060-001 - PE

Email: gillienecosta@hotmail.com 